\title{
Presencia de anticuerpos IgG anti-Toxoplasma gondii en embarazadas residentes en la Ciudad de la Habana.
}

\author{
Comunicación Breve
}

Carmen Acosta-Bas ${ }^{1}$, Xiomara Pérez ${ }^{1}$, Raquel García ${ }^{2}$.

${ }^{1}$ Centro de Inmunoensayo. Cubanacán, Ciudad Habana, Cuba. ${ }^{2}$ Policlínico 27 de noviembre, Marianao, Ciudad Habana, Cuba.

\section{RESUMEN.}

Se realizó un estudio seroepidemiológico en 207 gestantes, residentes en el municipio Marianao, de Ciudad de la Habana, con la finalidad de conocer la prevalencia de anticuerpos IgG anti-Toxoplasma gondii, sus concentraciones, expresadas en $\mathrm{UI} / \mathrm{mL}$ y las seroconversiones. El $60.3 \%$ de las gestantes resultaron positivas a $T$. gondii. El 24,6\%, del total de las encontradas positivas, presentan concentraciones de anticuerpos específicos superiores a $100 \mathrm{UI} / \mathrm{mL}$. Se sugiere la valoración de aspectos fundamentales relacionados con esta infección parasitaria dentro del Programa Nacional de Atención a las Embarazadas.

(Rev Biomed 2001; 12:250-254)

Palabras clave: Toxoplasmosis, mujeres gestantes, epidemiología, Cuba.

\section{SUMMARY.}

Prevalence of IgG anti-toxoplasma gondii antibodies in pregnant women in Havana City, Cuba.

A seroepidemiological study of 207 pregnant women living in Marianao, Havana City, was carried out with the purpose of measuring concentrations and seroconversions of Toxoplasma gondii anti- IgG antibodies. $60.3 \%$ of the pregnant women appeared to be positive to T. gondii. In $24.6 \%$ of the positive ones specific antibody concentrations higher than $100 \mathrm{IU} / \mathrm{mL}$ were reported. This study underlines the necessity of considering some important issues concerning these parasitic infections within the National Antenatal Program. (Rev Biomed 2001; 12:250-254)

Key words: Toxoplasmosis, pregnant women, epidemiology, Cuba.

Solicitud de sobretiros: Lic. Carmen Acosta-Bas. Centro de Inmunoensayo, Calle 134 y Av. 25, Reparto Cubanacán, Apdo. Postal 6945, Playa, Ciudad Habana,Cuba. E-mail: iqmonoclo@cie.sld.cu 


\section{Acosta-Bas, X Pérez, Raquel García.}

\section{INTRODUCCIÓN.}

La infección toxoplásmica adquirida antes de la concepción confiere a la mujer inmunidad permanente contra el riesgo de portar un feto afectado con toxoplasmosis. Excepcionalmente, se ha observado en mujeres embarazadas con inmunodeficiencia o en mujeres inmunocompetentes, linfopatías toxoplásmica periconcepcional $(1,2)$.

La infección contraída durante el embarazo puede ser transmitida al feto durante la fase de parasitemina mediante el paso transplacentario directo del parásito.

El riesgo de la infección fetal varía con la edad gestacional y con las condiciones inmunológicas de la embarazada. El riesgo periconcepcional (hasta la $6^{\mathrm{a}}$ semana) del pase del parásito al producto es de aproximadamente 1-2 \%, aumentando a un 15$20 \%$ en el período comprendido entre la $7^{\mathrm{a}}$ y la semana 24, incrementándose gradualmente de la semana 25 hasta el termino del embarazo, hasta ser de un $75 \%$. El riesgo global es del 60\%(3).

El tipo de manifestación varía en relación con el momento de la infección fetal. En el primer trimestre el paso de Toxoplasma gondii (T. gondii) provoca regularmente aborto y daños graves al feto. En el segundo trimestre predominan lesiones compatibles con la vida pero funcionalmente graves: triada de Bamatter (hidrocefalia, calcificaciones endocraniales, corioretinitis), lesiones oculares y neurológicas.

De la semana 25 hasta el término del embarazo la consecuencia de la infección toxoplásmica son menores, dada a una mejor capacidad del feto de defenderse (incremento significativo del pase transplacentario de la IgG específica materna (4), mejor estructuración fetal), pero puede persistir el riesgo de secuelas neuro-psíquicas $(5,6)$.

En la población cubana la infección por $T$. gondii es frecuente y a juzgar por el número de informes, la presencia de anticuerpos específicos varía entre un 40-66 \% en dependencia del área geográfica analizada y muy especialmente del inmunodiagnóstico empleado(7). Entre los serodiagnósticos más comúnmente utilizados figuran la Reacción de Fijación del Complemento y la Inmunofluorescencia Indirecta, expresándose la positividad en "títulos".

En este trabajo consideraremos de especial interés determinar la concentración de anticuerpos IgG específicos a $T$. gondii, así como la determinación de la IgM específica empleando la Inmunofluorescencia Indirecta en embarazadas residentes en el municipio Marianao en la capital del país.

\section{MATERIALES Y MÉTODOS.}

Se colectaron 207 pares de sueros que resultaron negativos a VIH, AgsHB y VDRL, procedentes de una población gestante sana comprendidas entre el $1^{\circ}$ y $2^{\circ}$ trimestre de embarazo que acudieron al control prenatal en la policlínica "27 de Noviembre" en el municipio Marianao en Ciudad de la Habana.

Los sueros se guardaron a -20C durante un tiempo no mayor de dos meses antes de ser procesados en busca de anticuerpos IgG anti- $T$. gondii mediante la técnica ultramicroELISA (UMELISA)(8).

El UMELISA es un ensayo cuantitativo de tipo indirecto con una curva de calibración desde 12.5 hasta $200 \mathrm{UI} / \mathrm{mL}$. Para el recubrimiento de la fase sólida se emplea un antígeno soluble de $T$. gondii. Los sueros, diluidos 1:20, al presentar anticuerpos específicos forman un inmunocomplejo que es reconocido por una anti-IgG humana obtenida en carnero y conjugada con fosfatasa alcalina, revelándose la reacción con la adición del sustrato fluorigénico. La fluorescencia obtenida es proporcional a la concentración de anticuerpos presentes. En todos los pasos el volumen de reactivos empleado es de $10 \mu \mathrm{L}$.

Todos los sueros que presentaron seroconversión y aquellos que presentaron una concentración ${ }^{3} 200 \mathrm{UI} / \mathrm{mL}$ se analizaron por la técnica de inmunofluorescencia indirecta (IFI) en busca de anticuerpos IgM específicos (9). Estos

\section{Revista Biomédica}




\section{Anticuerpos antitoxoplasma en mujeres embarazadas.}

fueron evaluados sin tratamiento previo o adsorbidos por Staphylococcus aureus, cepa Cowan I, para remover la IgG.

\section{RESULTADOS Y DISCUSIÓN.}

El cuadro 1 nos pone de manifiesto que de los 207 pares de sueros evaluados $125(60,3 \%)$ resultaron positivos a la presencia de anticuerpos IgG anti- $T$. gondii. Anteriormente Blanco y colaboradores (10) encontraron un $62,1 \%$ de positividad en sueros de mujeres en el momento del parto.

$\mathrm{Al}$ analizar las concentraciones de anticuerpos específicos, expresados en $\mathrm{UI} / \mathrm{mL}$, entre las gestantes consideradas positivas vemos que el 59.2 $\%$ de las mismas se insertan desde una infección pasada con su simple secuela serológica hasta la de una posible fase incipiente. Generalmente en estas mujeres la infección por $T$. gondii ha tenido lugar meses e incluso años antes de la toma de las muestras de sangre y no se esperan cambios en sus tasas de anticuerpos anti-T. gondii.

El $40,8 \%$ presenta anticuerpos específicos entre $100->200 \mathrm{UI} / \mathrm{mL}$, pudiendo interpretarse esto como concentraciones de valores medios, siendo representantes de una fase probable de actividad. Esta situación es de consideración, pues el número de gestantes enmarcadas en esta fase (51/125), representan el $40,8 \%$ de las gestantes positivas a $T$. gondii y el 24,6\%,(51/207), del total de ellas.

Tabla 1

Presencia de anticuerpos IgG anti-Toxoplasma gondii en embarazadas.

\begin{tabular}{lrc}
\hline $\begin{array}{l}\text { Concentración } \\
\text { de anticuerpos } \\
(\mathrm{UI} / \mathrm{mL})\end{array}$ & Muestras (n) & $\begin{array}{c}\text { Porcentaje } \\
(\%)\end{array}$ \\
\hline$<12,5$ & 82 & 39,7 \\
$>12,5-<25$ & 9 & 7,2 \\
$>25-<50$ & 25 & 20 \\
$>50-<100$ & 40 & 32 \\
$>100-<200$ & 30 & 24 \\
$>200$ & 21 & 16,8 \\
\hline
\end{tabular}

En todos los casos se realizó la determinación de anticuerpos $\operatorname{IgG}$ específica en el segundo trimestre del embarazo, estableciéndose en tres casos un incremento en la concentración del segundo suero respecto al primero que varió entre 3,77 a 12,91 veces. De igual forma, se encontraron dos casos negativos, que al evaluar su segundo suero se observó una franca seroconversión. Esto indica que nos encontramos en la rama ascendente de la curva de expresión de la evolución de los anticuerpos específicos, lo que sería igual a una primoinfección.

López y colaboradores emplearon un modelo de predicción de los porcentajes de adquirir toxoplasmosis durante el embarazo, obtenida de la distribución por edades de la población total de mujeres embarazadas (30.936) en Ciudad de la Habana y de la estimación de la máxima verosimilitud de $\mathrm{r}$, determinado que el riesgo global es de 1,37\% (11).

En el cuadro 2 se recogen los resultados de los 5 casos donde el segundo suero correspondiente al segundo trimestre de embarazo presenta un incremento mayor de tres veces la concentración respecto al suero evaluado en el primer trimestre, obteniéndose un porcentaje de seroconversión del $2,4 \%$. La diferencia encontrada entre lo reportado anteriormente y nuestros resultados, bien pudiera estar influida por el número de muestras analizadas (207 vs 3.196) o tal como ha sido indicado por Foulon y col. (5), la causa pudiera ser el cambio en la fuerza de la infección por el tiempo.

Al evaluar los pares de sueros positivos a IgG específica (casos que seroconvirtieron), por la técnica de Inmunofluorescencia Indirecta para la determinación de anticuerpos IgM anti- $T$. gondii, encontramos que los mismos resultaron positivos (cuadro 2).

Todos ellos resultaron negativos a factor reumatoideo. Es conocido que la simultánea asociación de los anticuerpos IgG anti-T. gondii

Vol. 12/No. 4/Octubre-Diciembre, 2001 
C Acosta-Bas, X Pérez, Raquel García.

Tabla 2

Seroconversión de anticuerpos IgG anti-T.gondii en embarazadas.

\begin{tabular}{lllll}
\hline $\mathrm{N}^{\mathrm{o}}$ del caso & 1er trimestre $(\mathrm{UI} / \mathrm{mL})$ & 2do trimestre $(\mathrm{UI} / \mathrm{mL})$ & Incremento (Veces) & IgM \\
\hline 58 & 5 & 61 & 12,5 & $1: 32$ \\
189 & 6 & 90 & 15 & $1: 64$ \\
130 & 12 & 155 & 12,91 & $1: 128$ \\
99 & 25 & 184 & 7,35 & $1: 64$ \\
7 & 45 & 170 & 3,77 & $1: 32$ \\
\hline
\end{tabular}

con los del factor reumatoideo conlleva a dar una reacción falsa positiva en el test de Remington (12).

Según lo propuesto por Dunn (13) y Frenkel (14), esto representaría aproximadamente una tasa 3,2-4,8/1000 casos de toxoplasmosis congénita anual entre niños, sobre la base de una tasa de incidencia de 30-45\%. De estos niños infectados, el $15 \%$ deben presentar daños clínicos severos y un $19 \%$ adicional padecerán una enfermedad ligera.

Con vista a reducir los efectos dañinos que puede provocar esta antropozoonosis, el trabajo debe estar dirigido principalmente sobre aquella parte de la población femenina embarazada que evidencie pruebas serológicas negativas a $T$. gondii entre 8-12 semanas de gestación, precisándose que medidas profilácticas deben extremarse para limitar las posibilidades de infección primaria. De ahí, la necesidad de que el diagnóstico de anticuerpos específicos a $T$. gondii se realice tempranamente durante el período de gestación.

\section{REFERENCIAS.}

1.- Rose I. Morphology and diagnostics of human toxoplasmosis. General Diagnos Pathol 1997; 42:257-70.

2.- Hennequin C, Dureau P, N'Guyen L, Thulliez $P$, Gagelin B, Dufier JL. Congenital toxoplasmosis acquired from an immune woman. Ped Infect Dis J 1997; 16:75-7.

3.- Forestier F, Daffos F, Hohlfeld P, Lynch L. Les foetopathies infectieuses. Prévention, diagnostic prénatale, attitude practique. Press Med 1991; 20:144-54.
4.- Lawton RL. B-Cell Development. In: Fetal ans Neonatal Physiology. Vol.2 chapt. 143. Philadelphia: W.B.Saunders Co.1992.

5- Pujol M, Duhamel C, Herlecoviez M. Prenatal diagnosis of toxoplasmosis. Press Med 1999; 28:1579.

6.- Nagy S, Hayde M, Panzenbock B, Adlassnig KP, Pollak A. Diagnosis of toxoplasmosis during pregnancy: Computer-assisted interpretation of serological test results. Wiener-Klinische-Wochenschrift 1997; 109:6416.

7.- Acosta C, Pérez X, Herrera R, Solis RL, López R, Machín R, et al. Evaluación clínica del UMELISAToxoplasma; Congreso "Biotecnología Habana 92"Cartel 19.15, 1992 Junio 8-12, 47.

8.- Remington JS, Miller MJ, and Brownlee I. IgM antibodies in acute Toxoplasma: I. Diagnostic significance in congenital cases and a method for their rapid demostration. Pedriatics 1968; 41:1082.

9.- Blanco R, Malberty A, López R, et al. Estudio de la presencia de anticuerpos contra Toxoplasma gondii en sueros de madres y recién nacidos en el momento del parto. Congreso 50. Aniversario del Instituto de Medicina Tropical Pedro Kouri. 1988. Resumen T-319:137-141.

10.- López R, Pérez X, Guerra R, Herrera R, Acosta Carmen. Toxoplasmosis entre mujeres embarazadas en Ciudad de la Habana. Biomédica 1993; 13:173-8.

11.- Foulon W, Naessens A, Lauwers S, et al. Impact or primary prevention on the incidence of toxoplama during pregnacy. Obstet Gynecol 1988; 72:363-6.

12.- Labro-Bryskler, Bryskler MT, López M, et al. Influence des facteurs rhumatoides sur la determination des anticorps antitoxoplasmes $\operatorname{IgM}$ par

\section{Revista Biomédica}




\section{Anticuerpos antitoxoplasma en mujeres embarazadas.}

inmunofluorescence et par agglutination. Ann Biol Clin 1981; 39: 175-8.

13.- Dunn D, Walon M, Peyron F, Petersen E, Peckhan C, Gelbert R. Mother-to-child transmission of toxoplasmosis: risk estimates for clinical counselling. Lancet 1999; 353:1829-33.

14.- Frenkel JK. Pathophysiology of Toxoplasmosis. Parasitol Today 1988; 4:273-8. 